\title{
Uncertainty and Resolution of Speckle Photography on Micro Samples
}

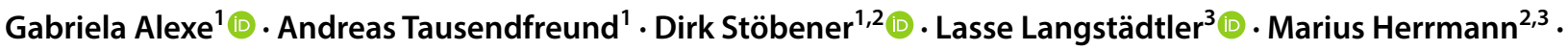 \\ Christian Schenck ${ }^{2,3} \cdot$ Andreas Fischer ${ }^{1,2}$
}

Received: 10 February 2020 / Revised: 28 February 2020 / Accepted: 2 March 2020 / Published online: 16 March 2020

(c) The Author(s) 2020

\begin{abstract}
The efficient development of new materials with defined properties requires fast methods of testing low volumes of material, such as a high-throughput investigation of spherical metallic micro samples with varying compositions and structuring treatments. A classical material testing method for macro samples, the tensile test cannot be employed for analyzing the mechanical properties of spherical samples with diameters below $1 \mathrm{~mm}$ since there are currently no methods for holding and stretching them. A combination between the incremental electrohydraulic extrusion as stress actuation unit and the speckle photography as strain measuring method is presented for obtaining the required mechanical characteristics. Positive longitudinal strain is generated at stepwise extrusion through $<1 \mathrm{~mm}$ wide forming channels using a liquid punch and the deformation is observed in situ after each forming step at the interface between the micro sample and a transparent window integrated into the forming die. The occurring local strain fields with a lateral extension down to $100 \mu \mathrm{m}$ and high gradients require displacement measurements with high lateral resolution over a large range of local dislocations between 0.1 and $>10 \mu \mathrm{m}$. It is unknown, whether the speckle strain measuring method is able to provide the necessary low uncertainty for the required resolution in the whole measuring range. Therefore, theoretical and experimental investigations of the deformation measurability using the speckle correlation method are presented, showing that local displacements up to $10 \mu \mathrm{m}$ can be measured with a spatial resolution between 3 and $10 \mu \mathrm{m}$ depending on the displacement size. The dominant effect influencing the measurement uncertainty for displacements at this high spatial resolution is the speckle noise, resulting into measurement uncertainties of less than $100 \mathrm{~nm}$. Hence, speckle photography is shown to be applicable for tensile test on micro samples.
\end{abstract}

Keywords Micro samples $\cdot$ Speckle photography $\cdot$ Electrohydraulic incremental micro forming

\section{Introduction}

The diversity of technological development requires a constant search for new materials with defined properties. In order to accelerate ample and expansive studies of various metal compositions and structuring treatments, a highthroughput investigation of spherical micro samples is

Gabriela Alexe

g.alexe@bimaq.de

1 Bremen Institute for Metrology, Automation and Quality Science (BIMAQ), University of Bremen, Linzer Str. 13, 28359 Bremen, Germany

2 MAPEX Center for Materials and Processes, University of Bremen, Postbox 330440, 28359 Bremen, Germany

3 Bremen Institute for Mechanical Engineering (bime), University of Bremen, Badgasteiner Straße 1, 28359 Bremen, Germany currently considered [1]. The investigation of micro samples results in descriptors, which are connected with the mechanical properties of the macro materials [2]. However, the classical material testing methods cannot be usually applied for spherical micro samples. Major adjustments of existing methods are necessary to accommodate for the dimensions and the geometry of the micro samples or novel methods must be found [2]. Specifically, the tensile test is a typical technique for the material characterization, which gives access to the average mechanical properties through the resulting strain states. The challenge of investigating the mechanical properties of micro samples consists on emulating the conventional testing processes: manipulating and holding the samples, applying and measuring small forces under uniaxial load, measuring the induced uniaxial strain fields.

Small-scale tensile methods are applied for nano and micro structures (microelectromechanical systems, thin 
films, nanowires, etc.) for investigating the deformation mechanisms at these length scales [3]. Depending on the material, dimension and type of structure, specific techniques are developed for every application. For example, a focused ion beam (FIB) system can be used for the production of dogbone tensile samples of less than $10 \mu \mathrm{m}$ in diameter and as actuation unit for the tensile testing accompanied by FIB/scanning electron microscopy imaging of the deformation [4]. However, there are currently no methods for holding and stretching spherical micro samples.

Changing the form of metallic samples in the micrometer range can be induced through micro forming [5]. Specific challenges of conventional forming are narrow tolerances necessary for the forming dies and samples, a precise guiding between punch and die as well as the requirement of a high stiffness of the mechanical punch. Therefore, the conventional forming methods are not suitable for economical high-throughput techniques. Electrohydraulic forming is an alternative especially for micro production out of sheet metal [6]. The impulse technology eliminates the fracture problem of high-aspect ratio punches and is rendering the setup more flexible to a varying geometry of die and sample. Electrohydraulic forming is already established in the macroscopic range and is used for reducing or expanding tubes and for processing sheet metal. First investigations regarding forming of bulk samples [7] and micro spheres [2] are reported.

Digital image correlation [8] (DIC) is an established optical method for full-field displacement measurements by correlating subimages of a surface after a small deformation with those of the undeformed surface. The ongoing development of the hardware, fast cameras, computers and algorithms allow the use of DIC for real time observation of dynamic processes [9]. The DIC technique is used for micro-scale (100-500 $\mu \mathrm{m})$ investigations of deformations in thin films [10] or serrated chips by cutting of titanium alloys [11]. However, this method depends on the existence of detectable features on the surface, naturally occurring (for example, texture of titanium alloy) or introduced through mechanical patterning or paint spraying. Even though a contactless optical measuring method, DIC is therefore in most cases invasive. Moreover, the spatial resolution for deformation detection and strain calculation is limited by the surface feature dimensionality. Laser speckle photography [12] transgresses these limitations and is already used for tensile tests [13] as well as an in-process measuring method for the induced deformation during machining $[14,15]$. Speckle photography is physically limited only by Heisenberg's uncertainty principle and the respective measurement uncertainty limit can be derived with the Cramér-Rao bound $[16,17]$. It has therefore the potential for precise small-scale measurements. However, applications for deformation/strain investigations at bulk forming of micro samples are not known.

The principle of emulating the tensile test for spherical micro samples was previously introduced as the combination between the incremental electrohydraulic extrusion as actuation unit and the digital image/speckle correlation (DISC) as strain measuring method [2]. Uniaxial loading of the micro samples is realized by extrusion into deep dies with different forming stages that represent defined stress and strain loads. The speckle patterns have to be measured in situ between the forming steps in order to follow the strain history, which requires an optical access to the process. Measuring the occurring displacement fields in situ with DISC limits the extrusion steps to several $10 \mu \mathrm{m}$ in order to avoid a strong speckle decorrelation [18]. Moreover, the impulse technique leads to strong vibrations in the actuation and measuring system and therefore to additional speckle decorrelation sources such as speckle contrast loss and defocusing. The following questions arise: Can uniaxial tensile strain be generated in the extruded micro sample? Are the induced displacements measurable with a DISC method? Can a setup provide the necessary spatial resolution and measurement uncertainty for displacements?

In this paper we investigate the measurability through DISC of the deformation induced at micro-extrusion. After describing the proposed measurement principle in Sect. 2, the measurement requirements and the actuation/measurement setup are defined in Sect. 3. The measurement uncertainty for displacements is theoretically assessed in Sect. 4 through Monte Carlo simulations of the speckle pattern measurements and the DISC evaluation. The contributions of the photon shot noise, camera noise and speckle noise are presented depending on the resolution of the evaluation. Moreover, other sources of speckle decorrelation due to the measurement setup and the process characteristics are identified and their influence on the measurement uncertainty is estimated. Section 5 comprises experimental validation of the theoretical results and experimental estimation of the measurement uncertainty due to defocusing. The discussion in Sect. 6 aims to define the optimal parameters of the measurement system, followed by conclusions in Sect. 7 .

\section{Principle of the Micro-Extrusion Strain Measurement}

Electrohydraulic extrusion is adapted as a micro-forming method which generates regions with uniaxial tensile and compressive strain. These strain fields are measured via an inspection window with DISC as an in situ measurement method, see Fig. 1. 


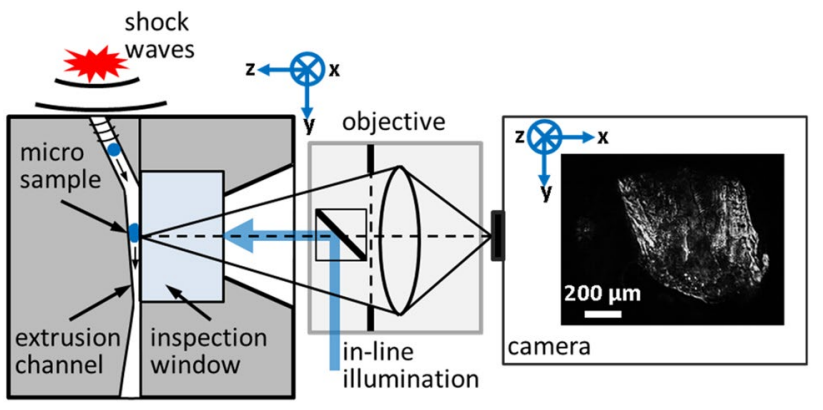

Fig. 1 Schema of the testing set-up with a forming die for the extrusion of spherical samples (left) and the in situ speckle measurement (right)

\subsection{Electrohydraulic Incremental Micro Forming}

In the electrohydraulic incremental forming the mechanical punch is replaced by a shock wave which allows the extrusion of the micro samples through high aspect ratio channels (Fig. 1, left side). One approach to generate a shock wave is to explode a wire in a water filled pressure chamber. The required energy is provided by the electrical discharge of a current pulse generator and the resulting reactions in the arising plasma. For the explosion a minimum energy is required, determined by the material, diameter and length of the wire. The resulting shock waves transmitting through water cause a pressure increase up to several giga pascal and convey the punching force within a few microseconds. The shock wave necessary for the extrusion is reached by increasing the loading energy above the vaporization energy of the wire. However, an incremental forming with small consecutive steps is required for the DISC measurements. The size of the extrusion steps is controlled through the shock wave energy by changing the loading energy of the pulse generator, adapted to the material, size and geometry of the sample and of the forming channel. Micro samples covering a wide formability range can be therefore analyzed without changing the extrusion tool by changing the exploding wire characteristics or the provided energy.

\subsection{Speckle Photography}

The functional principle of the image processing (the DISC evaluation) is shown in Fig. 2. The elastic deformations are determined by comparing speckle images of loaded and unloaded workpiece states. This comparison is performed locally by calculating the position of the maximum cross correlation in an evaluation window of size $W_{\text {eval }}$ in the field of view $(F O V)$. The local surface deformation in the image plane (for example the in-plane displacement in $x$-direction) is determined from the difference between the position of the cross correlation's maximum and the evaluation window center. In

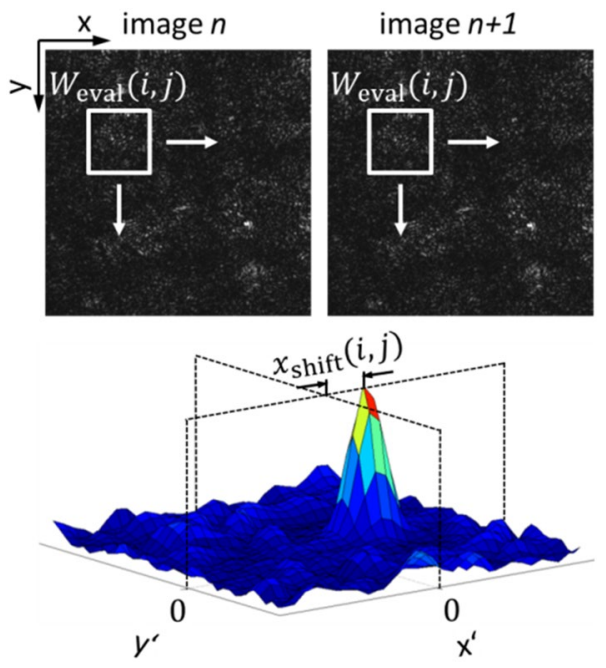

Fig. 2 Principle of DISC: the components $x_{\text {shift }}$ and $y_{\text {shift }}$ of the local displacement of the surface element $(i, j)$ are determined as the shift of the correlation maximum from the center of the $(i, j)$ evaluation window

order to obtain the global deformation field, the evaluation window scans with half-window wide steps across the whole field of view, whereby the calculated local displacements $x_{\text {shift }}, y_{\text {shift }}$ are stored for each scan position $(i, j)$. Due to the almost Gaussian profile of the cross-correlation function, a two-dimensional Gaussian curve is fitted to the correlation data to determine the maximum position with subpixel resolution $[19,20]$.

\subsubsection{Spatial Resolution of DISC}

For speckle photography the spatial resolution for the displacement distribution is defined as half of the evaluation window width $W_{\text {eval }}$ set for the cross-correlation calculations projected on the investigated surface.

$\Delta x=\frac{W_{\text {eval }}}{2} \frac{S_{\text {pixel }}}{|M|}$.

The evaluation window width, expressed in pixels in the image plane, is multiplied with the physical width of a detector pixel $s_{\text {pixel }}$ and divided by the absolute value of the magnification $M$ of the measurement optical system. The best achievable spatial resolution is however limited by the optical resolution, which is $0.61 \lambda / N A$ for a system with the numerical aperture $N A$ and illumination with the laser wavelength $\lambda$. With other words, the minimal reasonable evaluation window is approximately 2 times the size of a fully developed speckle $s_{\text {speckle }}$ [12] in the image plane:

$W_{\text {eval,min }}=2 \frac{0.61 * \lambda}{N A} \frac{|M|}{s_{\text {pixel }}} \approx 2 s_{\text {speckle }}$. 
Note that by the definition of Goodman [21] for speckles observed through a circular aperture, the speckle size

$s_{\text {speckle }}=\sqrt{\frac{1-N A^{\prime 2}}{\pi * N A^{\prime 2}}} \frac{\lambda}{s_{\text {pixel }}} \approx \frac{\lambda}{\sqrt{\pi} * N A} \frac{|M|}{s_{\text {pixel }}}$,

is $~ 92 \%$ of the Airy disk projected on the detector for systems with $N A \ll|M|$, with $N A^{\prime}=N A /|M|$ the numerical aperture on the image side of the optical system.

Improving the spatial resolution beyond $1 \mu \mathrm{m}$ means a numerical aperture $N A \geq 0.247$ for $\lambda=0.405 \mu \mathrm{m}$. Additional improvement of the spatial resolution through a decrease of the physical pixel size of the detector or increase of the imaging system magnification, as suggested by Eq. (1), occurs only as long as the required resolution is larger than the diffraction limit. Otherwise this leads to an increase of the speckle size and of the minimum evaluation window size $W_{\text {eval,min }}$, respectively. Note finally that the magnification is ultimately limited by the minimum required field of view $F O V$ reported to the sensor size $s_{\text {sensor }}$, i.e. $M_{\max }=s_{\text {sensor }} / F O V$.

\section{Measurement Setup and Requirements}

Both the forming process, in particular the geometry of the forming channel, and the optical measurement system must be designed in such a way that tensile strain can be generated and measured in a micro sample. Both areas are subject to individual restrictions and influence each other so that a common optimum must be found.

\subsection{Measurement Requirements}

Spherical metallic micro samples with diameters between $600 \mu \mathrm{m}$ and $1000 \mu \mathrm{m}$ are deformed in micro extrusion channels. Constant stress and strain conditions are desired independent of the sample diameter and sample material. Furthermore, manufacturing the micro channels with the required dimensions is a challenge. Finally, an optical access to the deformed sample is required for the in situ DISC measurements. For these reasons simulations of the process are performed to support the design of the extrusion channels which lead to uniaxial tensile strain.

\subsubsection{Forming Simulations}

A channel geometry which simplifies manufacturing and causes tensile strain fields over large areas independent of spheres diameter is a $\mathrm{V}$-groove with a constant reduction of width and depth along the extrusion path, see Fig. 3a. The flat inspection window acts as a part of the channel. Figure $3 b$ depicts exemplary results of 3D FEM simulations

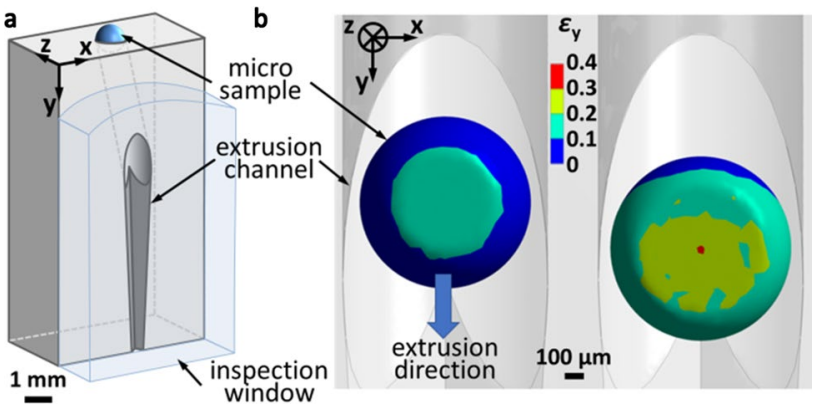

Fig. 3 Forming simulations: a V-groove forming channel; b 3D-simulation of one forming step of $\sim 300 \mu \mathrm{m}$ for an aluminum sphere with $900 \mu \mathrm{m}$ diameter showing the longitudinal strain in the extrusion direction $\varepsilon_{\mathrm{y}}($ in $\mu \mathrm{m} / \mu \mathrm{m})$

of the extrusion of an aluminum microsphere in a V-groove channel with a $3 \%$ width reduction. A bilinear hardening model is assumed for the calculations with the software ANSYS. Positive longitudinal strain in the extrusion direction $\varepsilon_{\mathrm{y}}$ results after an extrusion step.

Investigation of strain fields with a lateral extension down to $100-200 \mu \mathrm{m}$-depending on sample dimensions and position in the extrusion channel-with relatively high gradients require a spatial resolution $\Delta x$ of less than $10 \mu \mathrm{m}$. Due to the incremental procedure the temporal resolution $\Delta t$ is not influencing the deformation measurability. While in the extrusion direction global displacements up to $100 \mu \mathrm{m}$ are induced, local displacements in the extrusion and in the lateral direction of only several $\mu \mathrm{m}$ or even less than $1 \mu \mathrm{m}$ have to be detected. Consequently, a measurement uncertainty for displacements $\sigma$ of less than $100 \mathrm{~nm}$ is needed.

\subsection{Extrusion Setup}

An oblique $1 \mathrm{~mm}$ wide cylindrical channel through the steel die leads the micro sample without modifications to the forming and measuring region. The extrusion channel is realized as a $\mathrm{V}$-groove with the width decreasing from $1 \mathrm{~mm}$ at the beginning of the inspection window to $0.5 \mathrm{~mm}$ over a length of $7 \mathrm{~mm}$. The optical access is realized by replacing a half of the channel wall with a transparent material which is not interfering with the forming process or impeding on the DISC measurement of the displacement fields at the sample surface. As low friction between the window surface and sample as well as a high dynamic stiffness is required, sapphire is used as window material. Forming tests performed on micro samples with different properties (for example, aluminum A199.5, steel 100Cr6) showed the ability of $10 \mathrm{~mm}$ thick sapphire to withstand even high forming energies, in contrast to borosilicate glass and pre-stressed glass. Less wear and no cracks were observed on the inspection window after the forming steps. 


\subsection{Optical Measurement}

Considering the symmetry of strain fields, minimum fields of view $F O V$ slightly larger than $500 \mu \mathrm{m}$ are necessary in order to detect the strain history over the entire length of the extrusion channel. The optical system is translated to follow the deformed micro sample across the inspection window without changing the illumination conditions. The $10 \mathrm{~mm}$ diameter inspection window consists of $10 \mathrm{~mm}$ thick sapphire with a refraction index of 1.76. The accessible measurement region is maximized by orthogonally illuminating the sample surface. Therefore, a telecentric objective with inline illumination is used. The deployed objective VS-TM10-55CO (VS Technology Corporation) has $N A=0.23, M=10.5$ and a $55 \mathrm{~mm}$ working distance. Magnification $M=15.51$ is achieved by using a $4 \mathrm{~cm}$ extension tube. Illuminating with $\lambda=0.405 \mu \mathrm{m}$ laser light, a maximal spatial resolution $\Delta x=1.074 \mu \mathrm{m}$ is possible. Two cameras are used alternatively for this setup: the camera CP70 of the company Optronis GmbH (full well capacity FWC $=12300 \mathrm{e}^{-}, s_{\text {pixel }}=5.5 \mu \mathrm{m}$, resolution of $4080 \times 3072$ pixel $^{2}$ ) and the camera DX $4-285 \mathrm{FW}$ of the company Kappa optronics $\mathrm{GmbH}\left(\mathrm{FWC}=23000 \mathrm{e}^{-}\right.$, $s_{\text {pixel }}=6.45 \mu \mathrm{m}$, resolution $\left.1392 \times 1040 \mathrm{pixel}^{2}\right)$.

\section{Measurement Uncertainty}

Simulations of the measurement and the evaluation process of DISC are employed to investigate the measurement uncertainty for displacements of the proposed system. Physical limitation of the optical measurement is the photon shot noise, while camera noise was identified in a previous work [14] as a dominant contribution to the measurement uncertainty. The uncertainty of the DISC evaluation is further influenced by speckle noise, i.e. the influence of the different speckle patterns for the observed surfaces on the cross correlation result. In order to analyze the three contributions to the measurement uncertainty separately, simulations are performed at first for zero displacement.

The shot noise and camera noise induce speckle decorrelation in the simulated speckle images, which is evaluated as non-zero displacement. Speckle noise, however, cannot be estimated in the absence of displacements and other sources of noise since it does not change the speckle patterns when evaluating the cross-correlation of an image pair. Therefore, global shifts up to 3 pixel, in subpixel steps, are introduced in the simulated images before the DISC evaluation. For each type of noise, the measurement uncertainty for displacements $\sigma$ is determined as the standard deviation of the calculated displacements.

Speckle patterns with the size of $40 \times 40$ evaluation windows with statistically varying speckle contrast were numerically generated for speckle sizes up to $s_{\text {speckle }}=10$ pixel. The pseudo-random normally distributed phasor matrices which are used for the simulations lead to patterns of fully developed speckle [21]. Averaging over the large number of windows reduces the influence of the speckle noise on the calculated measurement uncertainty due to shot noise and camera noise. The speckle images were normed to the full well capacity $\mathrm{FWC}=12300 \mathrm{e}^{-}$of the camera CP70, which was also used in the measurement setup (Sect. 3.3). With other words, the speckle patterns are normed to full illumination independent of illumination time. For zero displacement, each of these patterns was overlaid 40 times with Poisson distributed photon shot noise and camera noise, respectively. Camera noise is simulated as constant white Gaussian noise. The resulting differences between the induced and the calculated average displacements are mostly below $10^{-6}$ pixel or at least 2 orders of magnitude below the respective standard deviation, meaning that no systematical contribution was introduced to the measurement uncertainty through the simulation and evaluation procedure. This way, up to $40 \times 40 \times 40=64000$ measurement points for each speckle/evaluation window size are generated. Note that averaging over the ensemble of simulated images means averaging over the speckle contrast. The limits of measurability and the measurement uncertainty budget presented in the next two subsections are evaluated for an average speckle contrast and for zero or 1D-displacements $y_{\text {shift }}$.

The displacement measurements are influenced also by the optical access and the process characteristics. Scattering at the inspection window interface to the material, volume scattering in sapphire or traces of water which was pressed by the shock wave between the window and the sample are phenomena leading to an overall contrast loss. The influence of the speckle contrast on the total measurement uncertainty is discussed in Sect. 4.3. As a result of extrusion, 2D strain fields are generated in the measurement plane $(x, y)$. Therefore, the influence of a displacement component $x_{\text {shift }}$ which is perpendicular to the evaluation direction on the measurement uncertainty of $y_{\text {shift }}$ is additionally theoretically estimated in Sect. 4.3. An important decorrelation source for this application is the defocusing $\left(z_{\text {shift }} \neq 0\right)$ in the optical system subjected to strong excitation of the setup during the extrusion. This effect cannot be simulated and will be treated experimentally in Sect. 5 after the validation of the theoretical considerations of Sect. 4.

\subsection{Limits of Measurability due to Photon Shot Noise}

For fully developed speckle patterns, the fundamental limit of the measurement uncertainty due to photon shot noise is expressed by the square root of the Cramér-Rao bound 
$C R B=\frac{1}{N_{\text {total }}} \frac{1}{4 \pi M^{2}} s_{\text {speckle }}^{2}$

where $N_{\text {total }}$ represents the total number of photons involved in the process [16]. The measurement uncertainty for an ideal speckle photography system is $\sigma_{0}=\sqrt{C R B}$, which is therefore proportional to the speckle size $s_{\text {speckle }}$. However, Eq. (4) is only valid for speckles larger than a pixel, since it does not consider pixel quantization effects. Moreover, it is valid for evaluation windows with a large number of speckles. With this conditions, the total number of photons $N_{\text {total }}$ is expected to vary proportional to the area of the evaluation window $W_{\text {eval }}^{2}$ or, for a given speckle size, to the average number of speckles evaluated as $N_{\text {speckle }}=\left(W_{\text {eval }} / s_{\text {speckle }}\right)^{2}$. This behavior is illustrated in Fig. 4 for speckles with $s_{\text {speckle }}=5$ pixel, where $N_{\text {total }}$ is calculated as the average number of photons illuminating each of the simulated speckle images which were overlaid only with Poisson distributed noise and considering a quantum efficiency of 1. The measurement uncertainty $\sigma_{0}$ varies linearly with $N_{\text {speckle }}$ in a double logarithmic representation, with a slope of $-1 / 2$ (dashed line) for $N_{\text {speckle }}>10$. The obtained deviation of $\sigma_{0}$ from this trend for $N_{\text {speckle }}<10$ is fully in agreement with the findings in [16].

Additionally, the measurement uncertainty due to the photon shot noise $\sigma_{\text {shot }}$ is calculated from the speckle decorrelation of the simulated speckle images, as described in the $2^{\text {nd }}$ paragraph of Sect. 4 . The difference between the empirical $\sigma_{\text {shot }}$ and the analytical $\sigma_{0}$, as seen in Fig. 4 , is due to averaging over many patterns with statistically varying

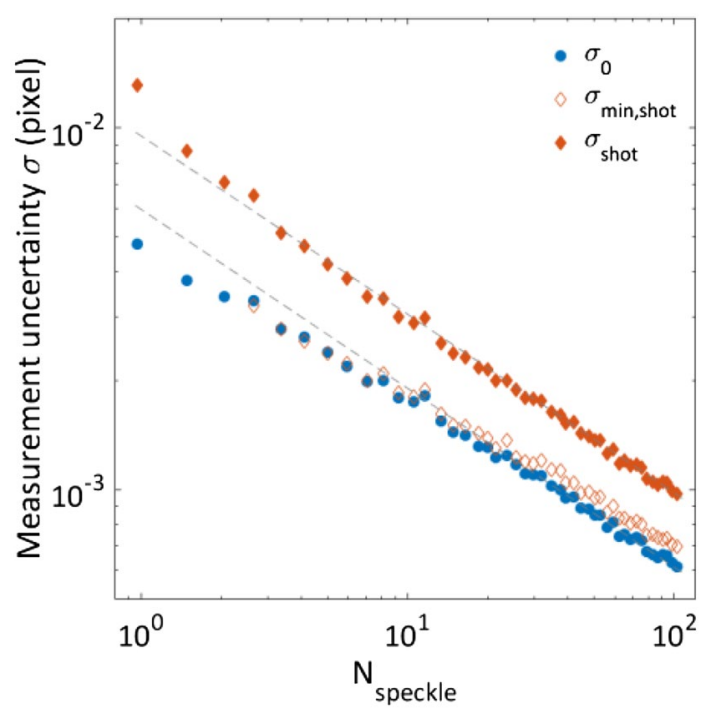

Fig. 4 Measurement uncertainty $\sigma_{0}$ due to $C R B$ and $\sigma_{\text {shot }}$ as a function of the average number of speckles $N_{\text {speckle }}$ in the evaluation window $W_{\text {eval }}$ for an average speckle size $s_{\text {speckle }}=5$ pixel; comparison to the minimum uncertainty due to shot noise $\sigma_{\min , \text { shot }}$. The dashed grey lines have a slope of $-1 / 2$ speckle contrast. The minimum value of the measurement uncertainty, an average over the lowest 10 values of the measurement uncertainty due to the photon shot noise, is $\sigma_{\text {min,shot }} \approx \sigma_{0}$ as expected since the $C R B$ describes the lower limit of measurability. Note that the $C R B$ is calculated using average values for $N_{\text {total }}$, which explains the values of $\sigma_{\text {min,shot }}<\sigma_{0}$.

For $N_{\text {speckle }}>4, \sigma_{\text {shot }}$ is also proportional to $\sqrt{N_{\text {speckle }}}$. The deviation from this trend of $\sigma_{\text {shot }}$ at $N_{\text {speckle }}<4$ roots in the cross-correlation algorithm. The cross-correlation peak loses in amplitude and sharpness and finding the position of the maximum is subject to a larger uncertainty. Therefore, an increase at small $N_{\text {speckle }}$ is expected for all components of the measurement uncertainty. Moreover, with only a few speckles in the window, the influence of the speckle noise is enhanced. Note, however, that reducing the size of the evaluation window below $2 s_{\text {speckle }}$ [cf. Eq. (2)], which means $N_{\text {speckle }}<4$, does not lead to an increase in spatial resolution and, thus, is not applied here.

\subsection{Measurement Uncertainty due to Speckle Noise}

Increasing the spatial resolution for the displacement measurements by choosing a small evaluation window leads to a reduced number of speckles that contribute to the correlation. The influence of the average number of speckles $N_{\text {speckle }}$ on the measurement uncertainty $\sigma_{\text {speckle }}$ (due to speckle noise) is shown in Fig. 5 over different displacements for the case of $s_{\text {speckle }}=5$ pixel. The measurement uncertainty $\sigma_{\text {speckle }}$ is determined as the standard deviation of the calculated displacements for $100 \times 100$ evaluation windows for each $N_{\text {speckle }}$ between $2^{2}$ and $10^{2}$. For full-pixel

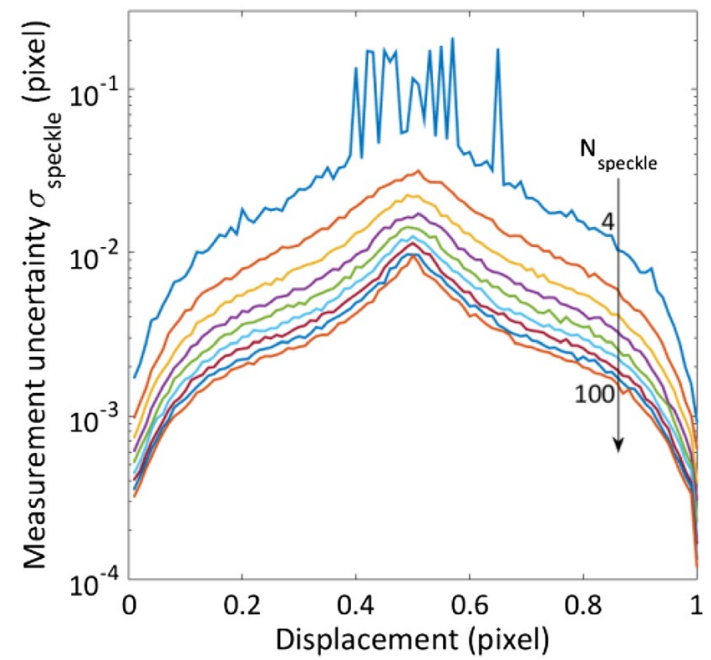

Fig. 5 Measurement uncertainty $\sigma_{\text {speckle }}$ as a function of the displacement $y_{\text {shift }}$ for an average speckle size $s_{\text {speckle }}=5$ pixel. The variation of the average number of speckles $N_{\text {speckle }}$ in the evaluation window $W_{\text {eval }}$ is indicated 
displacements, the cross-correlation algorithm reproduces exactly the displacement due to the absence of other sources of noise and the measurement uncertainty is zero. For subpixel displacements, the maximum of the cross-correlation peak is found through a 3 pixel $\times 3$ pixel Gaussian fit around the pixel with the highest value. Therefore the results of the subpixel interpolation are subject to a larger variance as the shift of the peak approaches half a pixel, which causes the highest asymmetry of the $3 \times 3$ matrix around the cross-correlation maximum. The effect of this asymmetry is strongly enhanced for the broad cross-correlation peaks which occur at $N_{\text {speckle }} \leq 4$, as can be observed in Fig. 5. Note that this behavior of speckle noise repeats itself for each subsequent full-pixel interval of displacements (not shown here).

\subsection{Measurement Uncertainty Budget}

Figure 6 shows the influence of $N_{\text {speckle }}$ on the measurement uncertainty due to the shot, camera and speckle noise at its maximum, for half a pixel displacements, cf. Figure 5. The total measurement uncertainty $\sigma_{\text {total }}$ results from the shot noise $\sigma_{\text {shot }}$, camera noise $\sigma_{\text {camera }}$, and the speckle noise $\sigma_{\text {speckle }}$ according to the propagation $\sigma_{\text {total }}=\left(\sigma_{\text {shot }}^{2}+\sigma_{\text {camera }}^{2}+\sigma_{\text {speckle }}^{2}\right)^{0.5}$. For displacements in the range of half a pixel $\left(y_{\text {shift }}=0.208 \mu \mathrm{m}\right.$, for a camera with $s_{\text {pixel }}=6.45 \mu \mathrm{m}$ and $\left.M=15.51\right), \sigma_{\text {speckle }}$ dominates the measurement uncertainty over the whole simulated range of $N_{\text {speckle }}$. For displacements $<0.3$ pixel and $N_{\text {speckle }}>4$, however, the total measurement uncertainty is

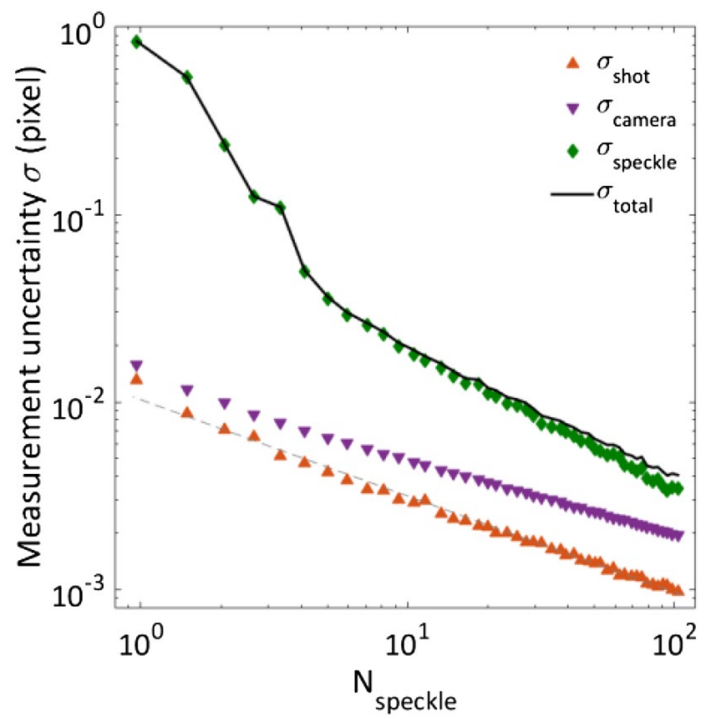

Fig. 6 Measurement uncertainty as a function of the average number of speckles $N_{\text {speckle }}$ in the evaluation window $W_{\text {eval }}$ for an average speckle size $s_{\text {speckle }}=5$ pixel. $\sigma_{\text {speckle }}$ is evaluated for a displacement $y_{\text {shift }}=0.5$ pixel dominated by $\sigma_{\text {camera }}$ (not shown in Fig. 6). At very small numbers of speckle in the evaluation window $\left(N_{\text {speckle }}<4\right)$ $\sigma_{\text {speckle }}$ is again the major component of the measurement uncertainty, independent of the displacement size.

Figure 7 shows the influence of the speckle size on the total measurement uncertainty for different average $N_{\text {speckle }}$ (grey curves). For $s_{\text {speckle }} \gtrsim 4$ pixel, $\sigma_{\text {total }}$ depends mainly on $N_{\text {speckle }}$. Below this value, quantization effects diminish the efficiency of the correlation algorithm. However, for small evaluation windows, an optimum between the effects of $s_{\text {speckle }}$ and $N_{\text {speckle }}$ must be found. For illustration, Fig. 7 also depicts the isocurves for $W_{\text {eval }}=30$ pixel, 20 pixel and 10 pixel, respectively. The measurement uncertainty displays an increase for $s_{\text {speckle }}$ below 2 pixel due to undersampling. Note that the cross-correlation algorithm of DISC does not work for $s_{\text {speckle }}<1$ pixel. The minimum uncertainty is reached between 2 and 3 pixel and increases with increasing $s_{\text {speckle }}$, which agrees with the findings in [14].

\subsection{Decorrelation}

An important aspect influencing the measurement uncertainty is the speckle contrast, which is usually defined as $C=\sigma_{I} / \bar{I}$, the ratio between the standard deviation and the average value of the intensity in the image [21]. This definition of contrast is however valid only for large images [22] and is therefore not applicable for small evaluation windows. A more relevant parameter for DISC is the cross-correlation coefficient $\gamma$, which is also influenced by the deviation of intensity from the expected (average) value [21]. A decorrelation factor $\delta$ according to [23] is defined as

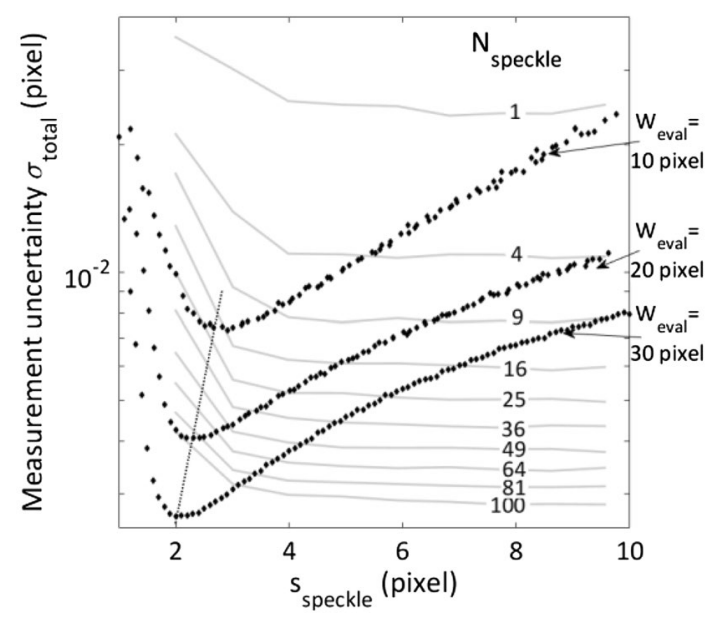

Fig. 7 Measurement uncertainty $\sigma_{\text {total }}$ as a function of the average speckle size $s_{\text {speckle }}$. Grey: isocurves for different average $N_{\text {speckle }}$. Black: curves for different $W_{\text {eval }}$ (10 pixel, 20 pixel and 30 pixel); the dotted line shows the tendency of the optimal $s_{\text {speckle }}$ for different $W_{\text {eval }}$ 
$\delta=\sqrt{\frac{1}{\gamma}-1}$.

The influence of speckle decorrelation on the measurement uncertainty $\sigma_{\text {total }}$ is shown exemplarily in Fig. 8 for $W_{\text {eval }}=10$ pixel and $s_{\text {speckle }}=4$ pixel, which means an average $N_{\text {speckle }}=6.25$. For these results, speckle patterns with large variations of contrast were generated and global shifts $y_{\text {shift }}$ up to 2 pixel were induced. The images consisted of $100 \times 100$ evaluation windows, each image with different $W_{\text {eval }}$ and different $s_{\text {speckle }}$. Both the original and the shifted patterns were overlaid with shot and camera noise. For zero and full-pixel displacements (Fig. 8a) $\sigma_{\text {total }}$ depends linearly on $\delta$ with a slope which is not affected by the displacement value. For $s_{\text {speckle }} \geq 4$ pixel the slope decreases with increasing $N_{\text {speckle }}$, meaning that for evaluation over larger numbers of speckle, the measurement uncertainty is less influenced by decorrelation (not shown here). At $\delta=0$ the measurement

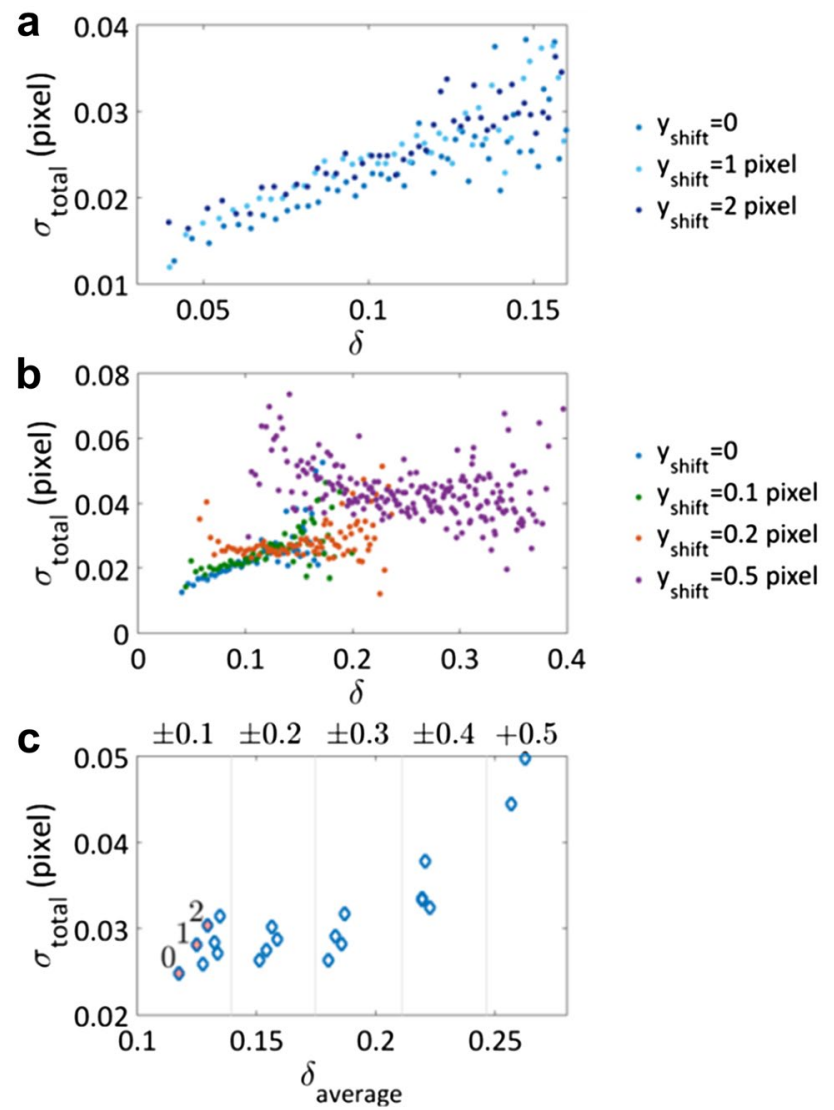

Fig. 8 Measurement uncertainty $\sigma_{\text {total }}$ as a function of the decorrelation factor $\delta$ for different displacements $y_{\text {shift }}$, indicated in pixel by the numbers in the graphs. An average speckle size $s_{\text {speckle }}=4$ pixel and $W_{\text {eval }}=10$ pixel $\left(N_{\text {speckle }}=6.25\right)$ is considered. a full-pixel displacements up to 2 pixel; $\mathbf{b}$ subpixel displacements up to 0.5 pixel; $\mathbf{c} \sigma_{\text {total }}$ versus the average $\delta$ for displacements up to 2 pixel in 0.1 pixel steps (full-pixel displacements are marked with red, the subpixel fraction is specified on top of the graph) uncertainty approaches $\sigma_{0}$, since this implies best correlation $(\gamma=1)$ and best contrast.

In the presence of subpixel displacements (Fig. 8b), the linear behavior of the measurement uncertainty with $\delta$ is only valid for very small displacements $y_{\text {shift }} \lesssim 0.1$ pixel. For subpixel displacements 0.2 pixel $<y_{\text {shift }} \leq 0.5$ pixel the effect of finite pixel size dominates the measurement uncertainty, which no longer depends on $\delta$ in the simulated range $(\delta \leq 0.4$ or $\gamma \geq 0.86$ ). This means that an improvement of the correlation beyond a certain limit does not lead to a significant decrease of the measurement uncertainty. A slight increase of the measurement uncertainty and $\delta_{\text {average }}$ with full-pixel value of $y_{\text {shift }}$ can be observed in Fig. 8c (red filled markers), a trend which is followed also for the corresponding subpixel $y_{\text {shift }}$. The variation of $\sigma_{\text {total }}$ versus $\delta_{\text {average }}$, respective versus $y_{\text {shift }}$, mirrors the variation of $\sigma_{\text {speckle }}$ (Fig. 5) combined with a displacement-proportional increase.

The extrusion process leads to large in-plane displacements at the interface to the inspection window. Therefore, the influence of large displacements $y_{\text {shift }}$ of several $\mu \mathrm{m}$ and of non-zero perpendicular components $x_{\text {shift }}$ on the speckle decorrelation and the measurement uncertainty for displacements has to be estimated. Figure 9a shows the overall increase of $\delta_{\text {average }}$ over a large range of displacements $y_{\text {shift }}$ considering also simultaneous perpendicular displacements $x_{\text {shift }}$. The decorrelation induced by the finite pixel size is enhanced in the presence of arbitrary oriented in-plane displacements. The increase of $\sigma_{\text {total }}$ with $y_{\text {shift }}$ is stronger than that of $\delta_{\text {average }}$, even for $x_{\text {shift }}=0$ (not shown here), because for some of the $10 \times 10$ pixel $^{2}$ evaluation windows

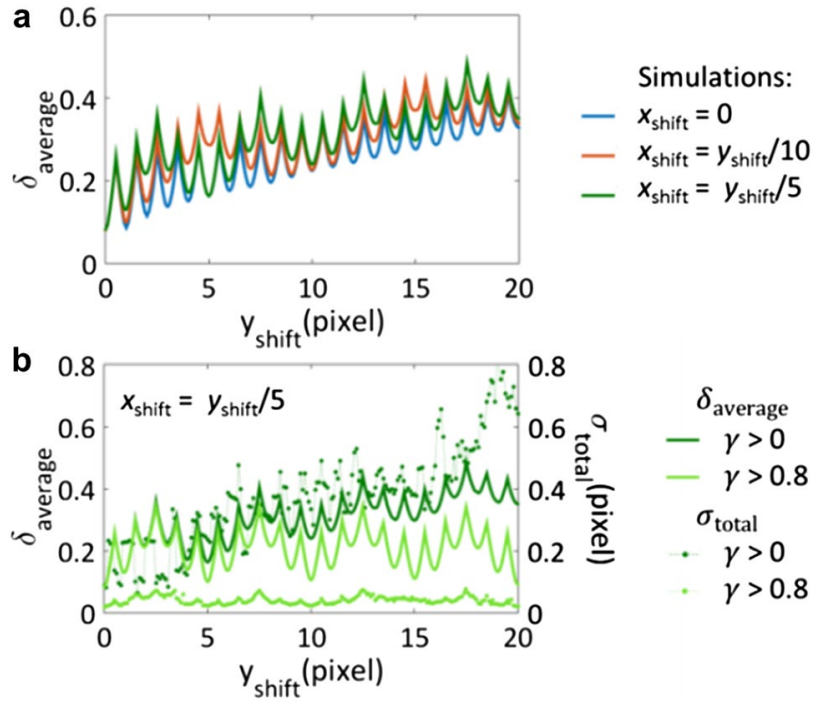

Fig. 9 a Average decorrelation factor $\delta_{\text {average }}$ versus the $y$-component $y_{\text {shift }}$ of a displacement with the $x$-component $x_{\text {shift }}=0, y_{\text {shift }} / 10$ and $y_{\text {shift }} / 5 ; \mathbf{b} \delta_{\text {average }}$ and measurement uncertainty $\sigma_{\text {total }}$ versus $y_{\text {shift }}$ for $x_{\text {shift }}=y_{\text {shift }} / 5$. An average speckle size $s_{\text {speckle }}=4$ pixel and $W_{\text {eval }}=10$ pixel $\left(N_{\text {speckle }}=6.25\right)$ is considered 
the correlation is completely lost $(\gamma \approx 0)$. Moreover, the measurement uncertainty $\sigma_{\text {total }}$ follows only tendentially $\delta_{\text {average }}$ for complex displacements, as shown in Fig. $9 \mathrm{~b}$ for the measurement uncertainty of the $y$-component $y_{\text {shift }}$ of a displacement with the $x$-component $x_{\text {shift }}=y_{\text {shift }} / 5$. Note that $\sigma_{\text {total }}$ is considered as the standard deviation of the calculated $y_{\text {shift }}$ over all the 10000 evaluation windows with a large contrast variation. However, an optimized speckle contrast leads to decreased measurement uncertainties. Selecting only the windows with the correlation coefficient $\gamma>0.8$ $(\delta<0.5)$ for the evaluation, not only that $\delta_{\text {average }}$ and $\sigma_{\text {total }}$ decrease, but they are also no longer significantly influenced by the full pixel value of $y_{\text {shift }}$. For evaluation windows with $N_{\text {speckle }}>6.25$, the resulting measurement uncertainty allows for displacement measurements with even lower correlation $\gamma$ than 0.8 , particularly for displacements of several pixels and larger.

For the example of a camera with a pixel width $s_{\text {pixel }}=6.45 \mu \mathrm{m}$ and an optical system with a magnification $M=15.51$, as also used in the experimental section, an image pixel corresponds to $0.416 \mu \mathrm{m}$. A $1 \mu \mathrm{m}$ displacement in $y$-direction can be therefore measured with a measurement uncertainty between $30 \mathrm{~nm}$ and $90 \mathrm{~nm}$ at a spatial resolution $\Delta x=2.1 \mu \mathrm{m}$, depending on the contrast. For comparison, for a camera with $s_{\text {pixel }}=3.45 \mu \mathrm{m}(1$ pixel $=0.222 \mu \mathrm{m})$ the measurement uncertainty for the same displacement lies between $16 \mathrm{~nm}$ and $50 \mathrm{~nm}$ at the same $\Delta x$ as above. The theoretical measurement uncertainty lies below the required $100 \mathrm{~nm}$. For displacements of several $\mu \mathrm{m} \sigma_{\text {total }}$ may reach larger values (up to $300 \mathrm{~nm}$ ). However, the relative measurement uncertainty remains below $5 \%$, which would be sufficient for the displacement measurements.

Constant illumination conditions are assumed for the simulated displacements. This assumption is not necessarily valid for larger deformations, which could lead to higher speckle decorrelation than estimated. Another important decorrelation source for this application is the defocusing in the optical system subjected to strong excitation of the setup during the extrusion. These effects cannot be accessed by simulations with reasonable efforts and will be treated experimentally in the next section, after validation measurements for the theoretical considerations concerning the measurement uncertainty.

\section{Experimental Results}

\subsection{Validation Measurements}

Figure 10 depicts the measurement uncertainty for displacements as resulting from DISC simulations $\sigma_{\text {total }}$ and from validation measurements $\sigma_{\text {measurement }}$ for zero displacements. For imaging, the camera CP70 $\left(\mathrm{FWC}=12300 \mathrm{e}^{-}\right.$,

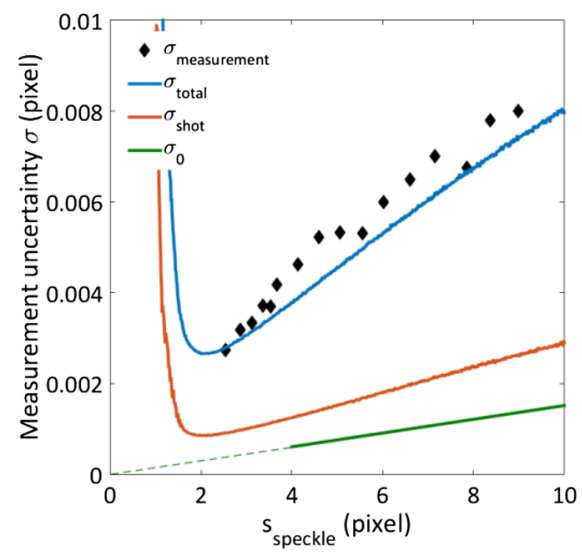

Fig. 10 Simulation and validation through measurements of a reference sample with an Optronis (CP70) camera $\left(\mathrm{FWC}=12300 \mathrm{e}^{-}\right)$and evaluated with $W_{\text {eval }}=30$ pixel

parameter also used for simulating the camera noise) and an imaging lens (Apo Rodagon) with adjustable aperture (f/4 to $\mathrm{f} / 22$ ) were employed. A laser with $\lambda=0.405 \mu \mathrm{m}$ illuminated the surface of a reference sample with a Gaussian beam profile at an incidence angle of about $30^{\circ}$. Speckle patterns with $s_{\text {speckle }}$ between 2 and 10 pixel were generated by varying the aperture. Both the simulated and the measured speckle patterns were evaluated with $W_{\text {eval }}=30$ pixel in order to increase the range of accessible speckle sizes. The measurement results correspond very well with the simulated data, for average decorrelation (considering all evaluation windows) and for lowest decorrelation (considering only ten windows with the highest value of $\gamma$, not shown in Fig. 10) as well. Note that the simulated data are also presented in Fig. 7. For completion, the measurement uncertainty $\sigma_{0}$ due to $C R B$ and $\sigma_{\text {shot }}$ as functions of the speckle size are shown.

The reference measurements were repeated with the camera DX4 - $285 \mathrm{FW}(\mathrm{FWC}=23000 \mathrm{e})$ and the telecentric objective with $N A=0.23$ and inline illumination ( $\lambda=0.405 \mu \mathrm{m}$ ). A magnification $M=15.51$ was achieved by using a $4 \mathrm{~cm}$ extension tube. The DISC evaluation of the measurements for both optical systems was realized with an evaluation window of 10 pixel. Figure 11 shows that for $s_{\text {speckle }}$ of about 4 pixel the linear behavior of the measurement uncertainty with the decorrelation $\delta$ is independent on the measurement optical system. Moreover, by comparing with the simulation results, an only slightly larger slope can be calculated for the measurements $\left(0.18\right.$ pixel $\left.^{-1}\right)$ than for simulations $\left(0.16\right.$ pixel $\left.^{-1}\right)$.

Thus, the estimated measurement uncertainty and its linear behavior with the speckle decorrelation is validated for zero displacement. Subsequently, the influence of large and 2D displacements on $\delta$ and $\sigma_{\text {total }}$ is experimentally investigated and compared with the theoretical results. 


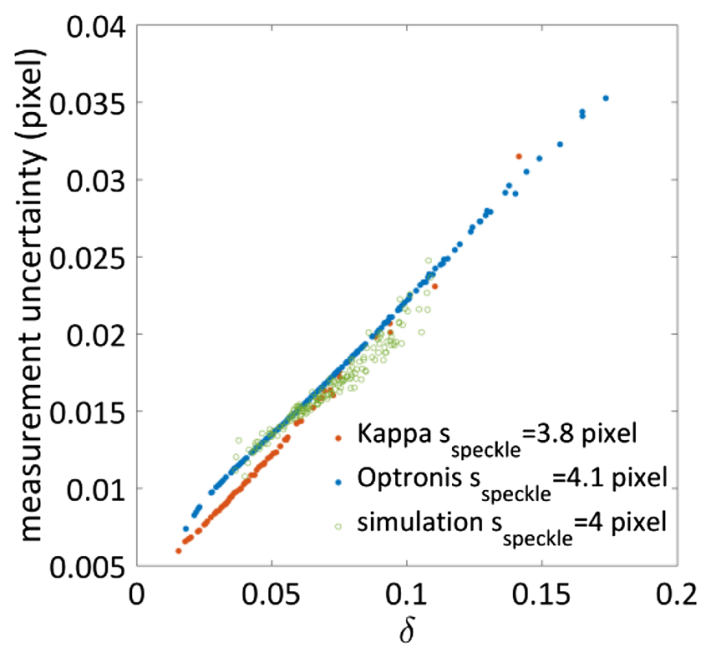

Fig. 11 Measurements with the CP70 (Optronis) camera $\left(\mathrm{FWC}=12300 \mathrm{e}^{-}, s_{\text {pixel }}=5.5 \mu \mathrm{m}\right)$ and $s_{\text {speckle }}=4.1$ pixel compared with the DX4 - $285 \mathrm{FW}$ (Kappa) camera ( $\mathrm{FWC}=23000 \mathrm{e}^{-}$, $s_{\text {pixel }}=6.45 \mu \mathrm{m}$ ) and $s_{\text {speckle }}=3.8$ pixel and simulations (Fig. 8a) with $s_{\text {speckle }}=4$ pixel; for both measurements and the simulation $W_{\text {eval }}=10$ pixel

\subsection{Displacement Measurements}

On a reference sample global in-plane displacements were induced using a P-611.3S NanoCube $x y z$-nanopositioning system (PI GmbH \& Co. KG). The measuring system consists of the DX4 $-285 \mathrm{FW}$ camera and the telecentric objective with $M=15.51$. Displacements in the $y$-direction up to $10 \mu \mathrm{m}$ in $0.1 \mu \mathrm{m}$ steps, meaning 0.24 pixel steps, were realized for three different $x$ positions at a $0.2 \mu \mathrm{m}$ interval.

The DISC evaluation showed that the displacements had an $x$-component $x_{\text {shift }} \approx y_{\text {shift }} / 12$ due to a slight misalignment between the $y$-axis of the NanoCube and that of the camera. This result is illustrated by the modulation of the average decorrelation factor (Fig. 12a), which is a consequence of the finite pixel size. For $W_{\text {eval }}=10$ pixel (spatial resolution $\Delta x=2.1 \mu \mathrm{m}$ ), $\delta_{\text {average }}$ for the measurements is slightly higher than that for simulations, indicating regions of the measured speckle patterns with lower correlation (and lower contrast) than realized by simulations. The influence of the speckle decorrelation on the measurement uncertainty of $y_{\text {shift }}$ is mirrored in Fig. $12 \mathrm{~b}$ by the strongly increased standard deviation especially for half-pixel $x_{\text {shift }}$ Since $N_{\text {speckle }}=6.6$ (for $s_{\text {speckle }}=3.9$ pixel), the behavior of the speckle noise (Fig. 5) is therefore reproduced. Selecting only the windows with the correlation coefficient $\gamma>0.8$ for the evaluation, the uncertainty is strongly reduced while still showing the same behavior (not shown here), rendering the measurement suitable for the application in the investigated displacement range. An alternative for reducing the measurement uncertainty is the resolution decrease. For comparison, a
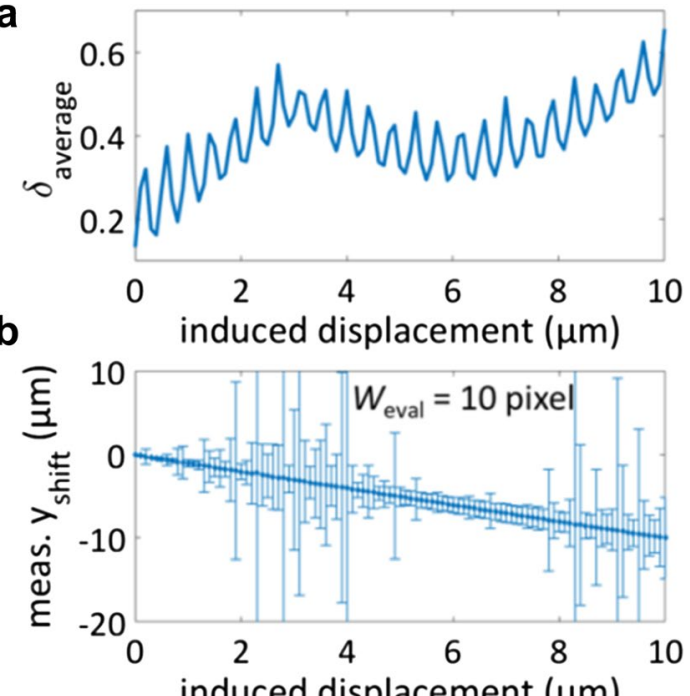

C

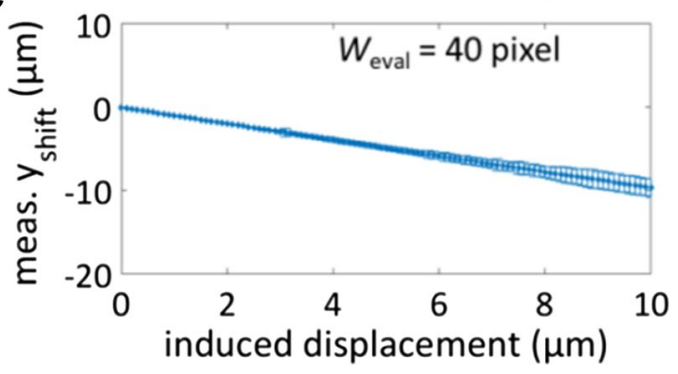

Fig. 12 a Average decorrelation factor $\delta_{\text {average }}$ versus the induced inplane displacements with components $x_{\text {shift }} \approx y_{\text {shift }} / 12$. Average of the measured $y$-displacements $y_{\text {shift }}$ and standard deviation for evaluation with $\mathbf{b} W_{\text {eval }}=10$ pixel and $\mathbf{c} W_{\text {eval }}=40$ pixel

the evaluation with $W_{\text {eval }}=40$ pixel (Fig. 12c) is no longer influenced by the finite pixel size and the speckle noise. The observed increase of the standard deviation is explained by the constant change of the optical way for each surface element contributing to the speckle formation, leading to a progressive change of the speckle pattern. For displacements $\gtrsim 9 \mu \mathrm{m}$, the relative measurement uncertainty is larger than $10 \%$ and therefore no longer suitable for our application. Note that the presented standard deviations mirror the uncertainty of a single measurement.

The influence of speckle decorrelation at high resolution is particularly relevant for subpixel displacements. Figure 13 shows the measurement uncertainty as a function of the decorrelation factor $\delta$ for different measured displacements $y_{\text {shift }}$ in comparison to the results of simulations (Fig. 8). The subpixel value of $y_{\text {shift }}$ determines the minimum achievable decorrelation. For $y_{\text {shift }}=0$, the expected linear behavior is observed, however with a slightly larger slope than resulting from simulations. Up to a threshold which is varying with the non-zero displacement value, the measurement uncertainty is not significantly influenced by the change of $\delta$. Beyond this threshold, $\sigma_{\text {total }}$ follows qualitatively the slope 


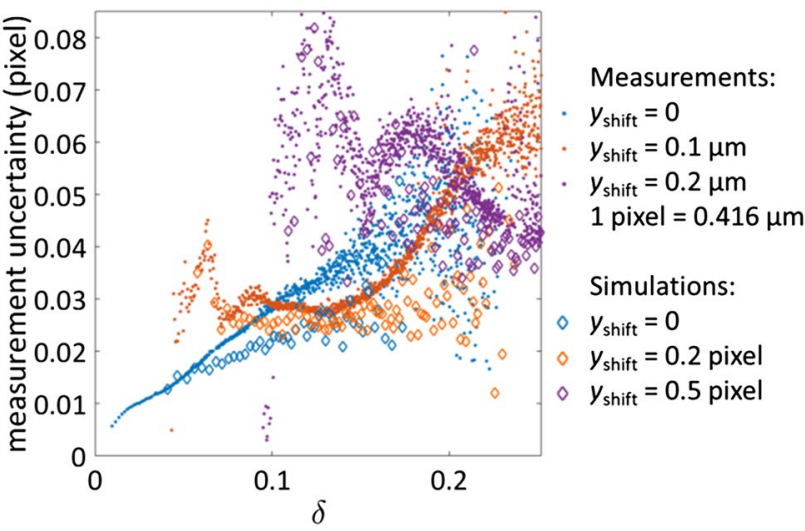

Fig. 13 Measurement uncertainty as a function of the decorrelation factor $\delta$ for different displacements $y_{\text {shift }}$. Measurements with the DX4 - 285 FW camera $\left(s_{\text {pixel }}=6.45 \mu \mathrm{m}\right)$ and $s_{\text {speckle }}=3.9$ pixel and simulations with $s_{\text {speckle }}=4$ pixel; for both measurements and the simulation $W_{\text {eval }}=10$ pixel

shown for $y_{\text {shift }}=0$. Therefore, the relative measurement uncertainty of $\lesssim 15 \%$ cannot be reduced only by improving the speckle correlation and a different approach for compensating the speckle noise must be found.

\subsection{Defocusing}

With the same measurement setup as described in the previous subsection, out-of-plane global shifts $z_{\text {shift }}$ of $\pm 10 \mu \mathrm{m}$, with $0.1 \mu \mathrm{m}$ steps, were also realized in order to investigate the influence of defocusing on the in-plane displacement measurement. Figure 14a shows the decorrelation effect of defocusing, at first for $y_{\text {shift }}=0$. The rapid increase of $\delta_{\text {average }}$ for $z_{\text {shift }} \neq 0$ is accompanied by a strong increase of the standard deviation for the evaluation of $y_{\text {shift }}$ (Fig. 14b) with $N_{\text {speckle }}=6.6$ $\left(W_{\text {eval }}=10\right.$ pixel, $s_{\text {speckle }}=3.9$ pixel). Moreover, a systematic effect of defocusing can be observed as the average measured $y_{\text {shift }}$ deviates significantly from zero beyond $z_{\text {shift }}= \pm 4 \mu \mathrm{m}$ $\left(y_{\text {shift }}=0.5 \mu \mathrm{m}\right.$ at $\left.z_{\text {shift }}= \pm 6 \mu \mathrm{m}\right)$. Speckle displacements can be expressed through a term directly proportional to object inplane displacements and a term proportional to the defocusing [24], which explains the systematic error. Note however that measurement uncertainties below $100 \mathrm{~nm}$ can only be reached in the range of negligible systematic error, region marked in the figure by the green rectangle. Considering only the windows with $\gamma>0.8$ for the cross-correlation evaluation diminishes the systematic error, but the measurement uncertainties do not decrease relevantly and the measurability region is therefore not enlarged (not shown here). The systematic influence of defocusing is reduced at lower spatial resolution, as shown in Fig. $14 \mathrm{c}$ for $W_{\text {eval }}=40$ pixel $\left(N_{\text {speckle }}=105.2\right)$. Additionally, the measurement uncertainties are also reduced leading to a larger measurability region. The so defined measurability region is determined by the measurement uncertainties
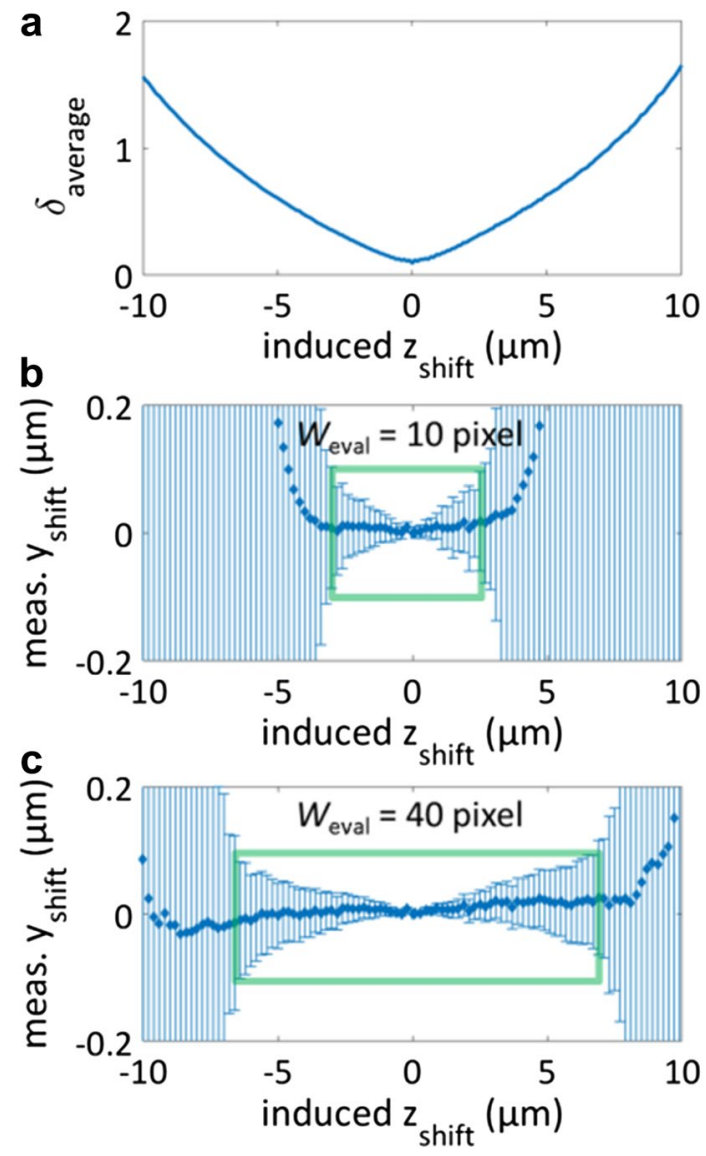

Fig. 14 a Average decorrelation factor $\delta_{\text {average }}$ versus the induced out-of-plane displacements $z_{\text {shift }}$ for $y_{\text {shift }}=0$. Average of the measured $y$-displacements $y_{\text {shift }}$ and standard deviation for evaluation with $\mathbf{b} W_{\text {eval }}=10$ pixel $\left(N_{\text {speckle }}=6.6\right)$ and $\mathbf{c} W_{\text {eval }}=40$ pixel $\left(N_{\text {speckle }}=105.2\right)$. Green rectangles mark the measurability region at zero displacement

due to defocusing in the presence of noise (shot, camera and speckle noise) at zero in-plane displacement. For non-zero (subpixel) displacements, the measurability region is smaller.

Defocusing with $1 \mu \mathrm{m}$ changes only slightly the variation of the measurement uncertainty with decorrelation, as illustrated in Fig. 15 for an in-plane displacement $y_{\text {shift }}=0.1 \mu \mathrm{m}$ ( 0.24 pixel). For $z_{\text {shift }}=2 \mu \mathrm{m}$, however, a relative measurement uncertainty up to $30 \%$ results, independent of the decorrelation factor. The measurability region is reduced by a factor of 2 as compared to Fig. 14b. With other words, at the spatial resolution $\Delta x=2.1 \mu \mathrm{m}$ the in-plane displacement measurement is very sensitive to defocusing.

\section{Discussion}

Simulations of the forming process showed that longitudinal tensile strain can be generated in micro samples at the incremental electrohydraulic extrusion depending on the shape of 


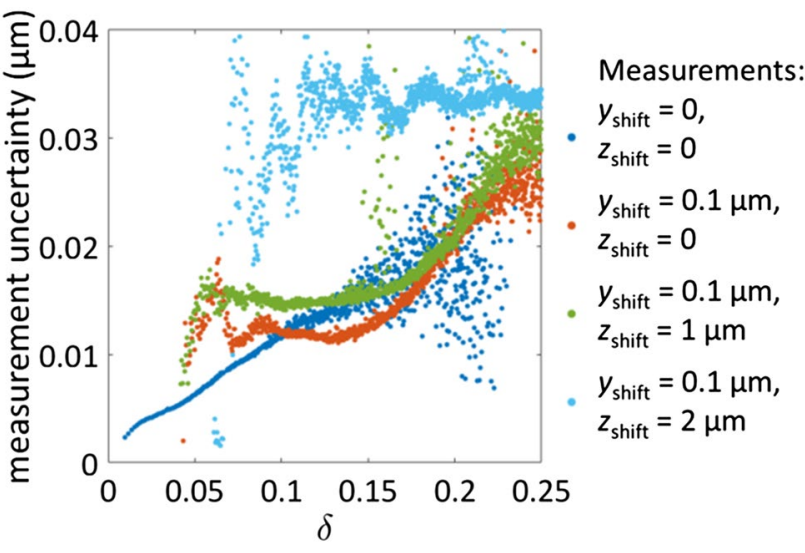

Fig. 15 Measurement uncertainty as a function of the decorrelation factor $\delta$ for different displacements $y_{\text {shift }}$ and different out-of-plane displacements $z_{\text {shift }}$. Measurements with the DX4 - 285 FW camera, $s_{\text {speckle }}=3.9$ pixel and $W_{\text {eval }}=10$ pixel

the extrusion channel. Furthermore, the displacement measurement requirements could be determined with the simulations (Sect. 3.1). In-plane displacements of from less than a $\mu \mathrm{m}$ to several $\mu \mathrm{m}$ have to be detected with a measurement uncertainty $\sigma<100 \mathrm{~nm}$ and a spatial resolution $\Delta x<10 \mu \mathrm{m}$. Theoretical and experimental investigations of the measurability of the resulting deformations with the DISC method, respectively its limitations, are presented in the Sects. 4 and 5. The questions left open are whether the proposed optical setup fulfils the described measurement requirements and which restrictions for the extrusion process result from the limitations of the measurement method.

As shown in the previous sections, the achievable spatial resolution is typically limited at $\Delta x \approx 3 \mu \mathrm{m}$ for defocusing below $1.5 \mu \mathrm{m}$ and in-plane maximal displacements of less than $10 \mu \mathrm{m}$. As the spatial resolution is not an issue for the sample translation at extrusion, global displacements of several tens of micrometers can be accurately measured from the speckle images and subtracted from the displacement field of the micro sample surface. However, the resulting global speckle decorrelation adds to that induced by the local displacements, cannot be compensated by the algorithm and impedes therefore the evaluation with high spatial resolution of the deformation. Measurements of the deformation with a spatial resolution $\Delta x \approx 10 \mu \mathrm{m}$ are less sensitive to the global displacements and can be realized with the present setup also for images with lower speckle correlation. A measurement strategy which is currently tested involves adjusting locally the spatial resolution of the displacement evaluation to the calculated correlation coefficient.

Measurement uncertainties below $100 \mathrm{~nm}$ at high spatial resolution can be reached with the chosen setup. Particularly for small displacements $\leq 200 \mathrm{~nm}$, however, the achievable spatial resolution has to be limited to $\Delta x \geq 6 \mu \mathrm{m}$ in order to provide relative measurement uncertainties $\leq 10 \%$. Therefore, the achievable spatial resolution of the displacement measurement is determined by the smallest detected displacements. Alternatively, the spatial resolution can be locally adjusted during the evaluation depending on the measured displacement.

\section{Conclusions}

The realization of the tensile test for spherical micro samples by the combination of the incremental electrohydraulic extrusion as actuation unit and the digital image/speckle correlation as deformation measuring method was investigated theoretically (through simulations of the forming process and of the speckle measurement) and experimentally. Particularly the measurability of the local displacements with a high spatial resolution was studied in detail to identify important sources for the measurement uncertainty. A dominant effect influencing the measurement uncertainty at high spatial resolutions $\Delta x \leq 6 \mu \mathrm{m}$ is the speckle noise. Regarding the required spatial resolution, the theoretical and experimental results demonstrate the feasibility of the deformation measurement approach. With the presented experimental setup, local displacements of less than $10 \mu \mathrm{m}$ induced by extrusion steps of $\approx 10 \mu \mathrm{m}$ (global displacements) can be measured with a spatial resolution $\Delta x \geq 3 \mu \mathrm{m}$. For the measurement of small displacements $(\leq 200 \mathrm{~nm})$ a decrease of the measurement uncertainty is needed, which can be realized by minimizing the influence of the speckle noise through averaging over different speckle patterns. As a next step, the characterized displacement measurement system for micro samples is applied for investigating the strain fields which are generated at extrusion depending on the mechanical properties of the micro samples.

Acknowledgements Open Access funding provided by Projekt DEAL. This research is funded by Deutsche Forschungsgemeinschaft (DFG, German Research Foundation)-project number 276397488-SFB 1232 and the support is gratefully acknowledged.

Open Access This article is licensed under a Creative Commons Attribution 4.0 International License, which permits use, sharing, adaptation, distribution and reproduction in any medium or format, as long as you give appropriate credit to the original author(s) and the source, provide a link to the Creative Commons licence, and indicate if changes were made. The images or other third party material in this article are included in the article's Creative Commons licence, unless indicated otherwise in a credit line to the material. If material is not included in the article's Creative Commons licence and your intended use is not permitted by statutory regulation or exceeds the permitted use, you will need to obtain permission directly from the copyright holder. To view a copy of this licence, visit http://creativecommons.org/licenses/by/4.0/. 


\section{References}

1. Ellendt N, Mädler L (2018) High-throughput exploration of evolutionary structural materials. HTM J Heat Treat Mat 73(1):3-12

2. Steinbacher M, Alexe G, Baune M, Bobrov I, Bösing I, Clausen B, Czotscher T, Epp J, Fischer A, Langstädtler L, Meyer D, Raj Menon S, Riemer O, Sonnenberg H, Thomann A, Toenjes A, Vollertsen F, Wielki N, Ellendt N (2019) Descriptors for high throughput in structural materials development. High-Throughput $8(4): 22$

3. Gianola DS, Eberl C (2009) Micro- and nanoscale tensile testing of materials. JOM 61(3):24-35

4. Fu Z, Jiang L, Wardini J, MacDonald B, Wen H, Xiong W, Zhang D, Zhou Y, Rupert T, Chen W, Lavernia E (2018) A high-entropy alloy with hierarchical nanoprecipitates and ultrahigh strength. Sci Adv 4:eaat 8712

5. Vollertsen F et al (2020) Cold micro metal forming, lecture notes in production engineering. Springer, Berlin

6. Sondur SR, Pawar SV, Salunke SA, Salunke SR, Patil SB (2015) A model for deformation in high energy rate micro forming process. Int J Adv Manuf Technol 76(1-4):189-197

7. Langstädtler L, Pegel H, Herrmann M, Schenck C, Kuhfuss B (2018) Electrohydraulic incremental bulk metal forming. MATEC Web Conf 190:03001

8. Sutton MA, McNeill SR, Helm JD, Chao YJ (2000) Advances in two-dimensional and three-dimensional computer vision. Photomechanics. Springer, Berlin (Germany), pp 323-372

9. Tarigopula V, Hopperstad O, Langseth M, Clausen A, Hild F (2008) A study of localisation in dual-phase high-strength steels under dynamic loading using digital image correlation and FE analysis. Int J Sol Struct 45:601-619

10. Eberl C, Gianola DS, Hemker KJ (2010) Mechanical characterization of coatings using microbeam bending and digital image correlation techniques. Exp Mech 50(1):85-97

11. Harzallah M, Pottir T, Gilblas R, Landon Y, Mousseigne M, Senatore $\mathrm{J}$ (2018) A coupled in situ measurement of temperature and kinematic fields in Ti-6Al-4V serrated chip formation at microscale. Int J Mach Tools Manuf 130-131:20-35

12. Ennos AE (1984) Speckle interferometry. In: Dainty JC (ed) Laser speckle and related phenomena, 2nd edn. Springer, Berlin, pp 203-253

13. Marth S, Häggblad H, Oldenburg M, Östlund R (2016) Post necking characterization for sheet metal materials using full field measurements. J Mater Process Tech 238:315-324

14. Tausendfreund A, Stöbener D, Fischer A (2018) Precise in-process strain measurements for the investigation of surface modification mechanisms. J Manuf Mater Process 9:1-11

15. Tausendfreund A, Stöbener D, Fischer A (2019) Induction of highly dynamic shock waves in machining processes with multiple loads and short tool impacts. Appl Sci 9(11):2293

16. Fischer A (2017) Fundamental uncertainty limit for speckle displacement measurements. Appl Opt 56:7013-7019

17. Fischer A (2019) Limiting uncertainty relations in laser-based measurements of position and velocity due to quantum shot noise. Entropy 21(3):264

18. Yamaguchi I (1981) Speckle displacement and decorrelation in the diffraction and image fields for small object deformation. Opt Acta 28:1359-1376

19. Zhou P, Goodson KE (2001) Subpixel displacement and deformation gradient measurement using digital image speckle correlation (DISC). Opt Eng 40:1613-1620

20. Nobach H, Honkanen M (2005) Two-dimensional Gaussian regression for sub-pixel displacement estimation in particle image velocimetry or particle position estimation in particle tracking velocimetry. Exp Fluids 38:511-515
21. Goodman JW (2010) Speckle phenomena in optics. Freeman, W. $\mathrm{H}$

22. Duncan DD, Kirkpatrick SJ, Wang RK (2008) Statistics of local speckle contrast. J Opt Soc Am A OSA 25:9-15

23. Sjödahl M (1997) Accuracy in electronic speckle photography. Appl Opt 36(13):2875-2885

24. Sjödahl M (1995) Calculation of speckle displacement, decorrelation, and object-point location in imaging systems. Appl Opt 34(34):7998-8010

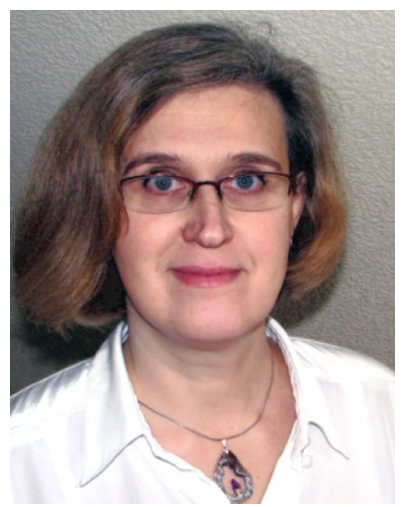

Gabriela Alexe studied physics at the University of Bucharest, Romania. At the Bremen Institute for Metrology, Automation, and Quality Science, where she holds a position as research assistant since 2015, she works in the research group "In-process metrology and optics". Her current research focuses on optical methods for the measurement of form and deformation of nanoand microstructures.

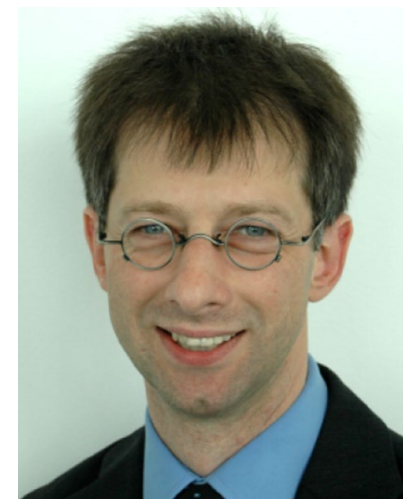

Andreas Tausendfreund studied physics at the University of Bremen and received his $\mathrm{PhD}$ in 2018 in the field of laser-optical measurement techniques for the characterization of surface defects on the nanometer scale. Since 2005 is he a research assistant at the BIMAQ and works in the research group "In-process metrology and optics". His work areas are the application of optical measurement techniques in manufacturing processes and the investigation and description of light scattering at nanostructures

based on Maxwell equations.

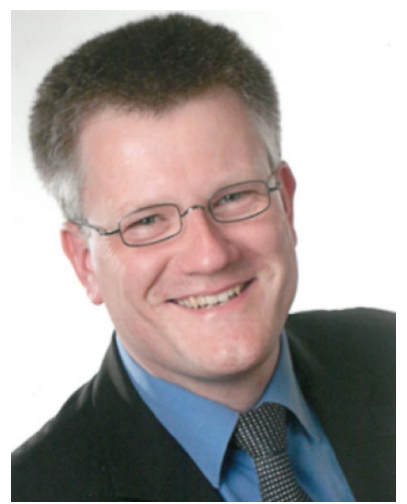

Dirk Stöbener (after studying physics) received his doctorate in 2016 at the University of Bremen in the field of production engineering. Since 2003 is he the head of the research group "Inprocess metrology and optics" at BIMAQ. His research interests include acoustic and optical methods for the productionrelated measurement of workpiece properties such as dimensions, deformation or roughness. 

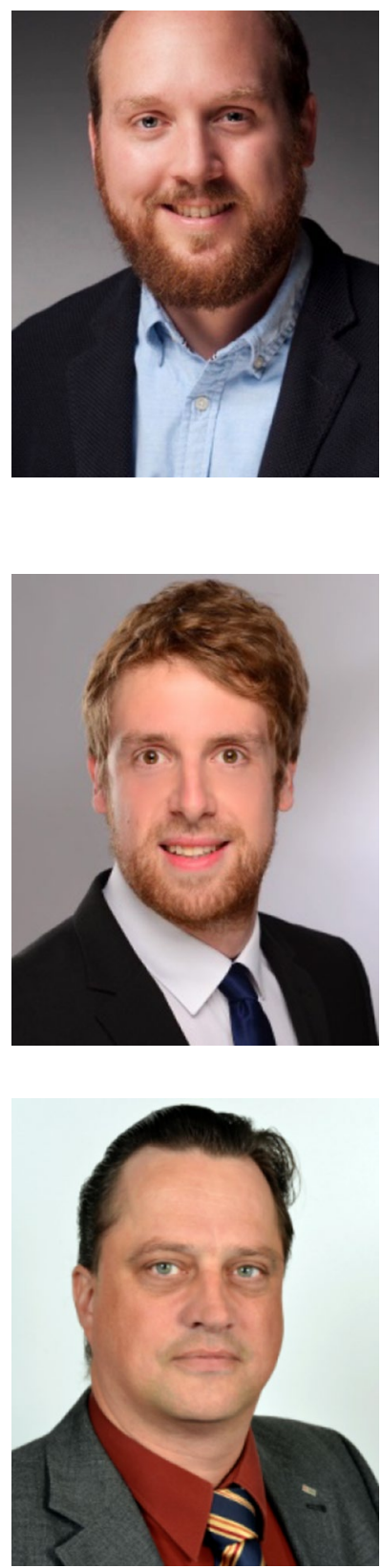

Christian Schenck received the $\mathrm{Ph}$.D. degree in mechanical engineering from the University of Bremen, Bremen, Germany, in 2008. Since then, he has been a Chief Engineer with the Bremen Institute for Mechanical Engineering, University of Bremen, and is the Head of the division mechatronics.

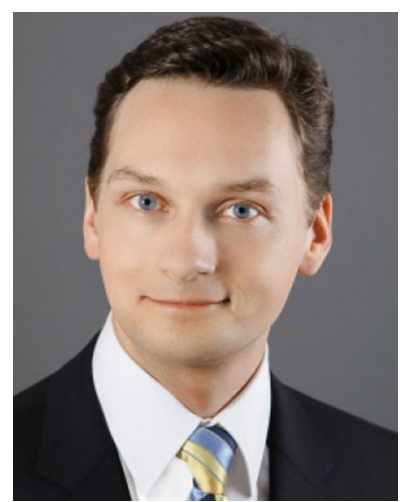

Andreas Fischer received the $\mathrm{Ph} . \mathrm{D}$. degree and the venia legendi degree in electrical engineering from Technische Universität Dresden, Dresden, Germany, in 2009 and 2013, respectively. From 2009 to 2016, he was the head of the group of measurement system techniques at the Laboratory for Measurement and Testing Techniques of the Technische Universität Dresden. Since 2016, he has been a Full Professor with the University of Bremen, Bremen, Germany, where he is the director of the Bremen Institute for Metrology, Automation, and Quality Science. His current research interests include the identification, characterization, and application of measurement limits as well as the development of optical measurement systems for investigating dynamic flow, shape, and surface phenomena. 